\title{
Insights into the structure and architecture of the CCR4-NOT complex
}

\author{
Kun Xu ${ }^{1,2}$, Yuwei Bai ${ }^{1}$, Aili Zhang 1,2, Qionglin Zhang ${ }^{2}$ and Mark G. Bartlam ${ }^{1,2}$ * \\ 'State Key Laboratory of Medicinal Chemical Biology, Nankai University, Tianjin, China \\ ${ }^{2}$ College of Life Sciences, Nankai University, Tianjin, China
}

\section{Edited by:}

Martine Anne Collart, University of

Geneva, Switzerland

\section{Reviewed by:}

Walter Lukiw, Louisiana State

University, USA

Sebastiaan Winkler, University of

Nottingham, UK

${ }^{*}$ Correspondence:

Mark G. Bartlam, College of Life

Sciences, Nankai University, 94 Weijin

Road, Tianjin 300071, China

e-mail: bartlam@nankai.edu.cn
The CCR4-NOT complex is a highly conserved, multifunctional machinery with a general role in controlling mRNA metabolism. It has been implicated in a number of different aspects of mRNA and protein expression, including mRNA degradation, transcription initiation and elongation, ubiquitination, and protein modification. The core CCR4-NOT complex is evolutionarily conserved and consists of at least three NOT proteins and two catalytic subunits. The L-shaped complex is characterized by two functional modules bound to the CNOT1/Not1 scaffold protein: the deadenylase or nuclease module containing two enzymes required for deadenylation, and the NOT module. In this review, we will summarize the currently available information regarding the three-dimensional structure and assembly of the CCR4-NOT complex, in order to provide insight into its roles in mRNA degradation and other biological processes.

Keywords: CCR4-NOT complex, mRNA decay, poly(A), deadenylation, NOT module, protein structure

\section{INTRODUCTION}

The CCR4-NOT is a highly conserved, multifunctional machinery with a general role in controlling mRNA metabolism (Collart and Panasenko, 2012). It catalyzes the deadenylation process, whereby the removal of mRNA poly(A) tails represses translation and marks the mRNA for degradation (Collart, 2003). Deadenylation, which is crucial for gene expression and is involved in biological processes ranging from embryonic development to learning and memory, is believed to be a biphasic process (Yamashita et al., 2005; Wahle and Winkler, 2013). The first phase involves synchronous and stepwise shortening of the poly(A) tail to $\sim 110$ nt. In the second phase, which is crucial for triggering decay of the mRNA body, the mRNAs become more heterogeneous in the lengths of their poly(A) tails, ranging from $\sim 20 \mathrm{nt}$ to $\sim 110 \mathrm{nt}$. Several observations support the hypothesis that biphasic deadenylation is the result of the sequential action of PAN2-PAN3 and CCR4-NOT complexes, with PAN2-PAN3 dominating the first phase and CCR4-NOT the second phase (Yamashita et al., 2005; Zheng et al., 2008; Wahle and Winkler, 2013). The CCR4-NOT complex is involved in a number of other important biological processes, including transcription initiation and elongation, ubiquitination and protein modification (reviewed in Collart and Panasenko, 2012). Members of the CCR4-NOT complex are also associated with various functions in several species, either in the nucleus or the cytoplasm, including DNA repair and histone methylation in yeast, spindle positioning, and regulation of microtubule length in Caenorhabditis elegans and spermatogenesis in mice.

The core CCR4-NOT complex is highly and evolutionarily conserved among eukaryotes (Draper et al., 1995; Albert et al., 2000; Gavin et al., 2002). Early studies on the Saccharomyces cerevisiae complex by mass spectrometry and co-immunoprecipitation led to the identification of the CCR4-NOT complex approximately
0.9-1.2 MDa in size (Liu et al., 1998). The yeast core complex was found to contain five NOT proteins (Not1-Not5) and two catalytic subunits, Ccr4 and Caf1 (Pop2). In mammals, including humans, their homologs also form a similar multi-subunit complex which plays a significant role in the regulation of several cellular machines (Mahadevan and Struhl, 1990; Collart and Struhl, 1993, 1994; Lee et al., 1998; Oberholzer and Collart, 1998; Badarinarayana et al., 2000; Albert et al., 2002; Deluen et al., 2002; Lenssen et al., 2002; Maillet and Collart, 2002; Viswanathan et al., 2003). Additional subunits in the human CCR4-NOT complex include RQCD1 (also known as CNOT9/Not9/Rcd1/Caf40), Caf130, Not10 (CNOT10), C2orf29 (CNOT11), and TAB182, although some are species specific. For instance, Caf130 is specific to yeast (Collart and Panasenko, 2012), while Not10, C2orf29, and TAB182 in the human complex are not found in yeast (Lau et al., 2009) and Not10 and C2orf29 are conserved in metazoans (Bawankar et al., 2013; Mauxion et al., 2013).

Interaction studies have shown the S. cerevisiae complex is centered on the scaffold protein Not1, which is essential for yeast viability (Maillet et al., 2000). Similarly, human CNOT1 has been shown to be essential for viability of cells (Ito et al., 2011b), and is required for cell proliferation but not cell viability in MCF7 cells (Mittal etal., 2011). Not4 was shown to possess a functional RING finger domain in its N-terminal by NMR (Hanzawa et al., 2001), and its human homolog was subsequently confirmed to function as an E3 ligase by in vitro ubiquitination (Albert et al., 2002). Not2 contains no known functional motifs but has two functional domains: a C-terminal region involved in CCR4NOT function, and an N-terminal domain that interacts with the protein Ada2 (Benson et al., 1998). Not3 and Not5 share similar N-terminal regions, but their respective functions remain unclear. The complex also contains two deadenylases (to be discussed in more detail below), as well as Caf40 and Caf130 with 
indeterminate function. A number of additional binding partners have been identified for the $S$. cerevisiae complex but will not be discussed further in this review (see Collart, 2003 for more details).

In contrast to the yeast CCR4-NOT complex, which is wellunderstood as a result of extensive yeast genetics studies, the human complex has been less well studied until relatively recently. Analysis of the human CCR4-NOT complex from stable HeLa cell derivatives expressing epitope-tagged CCR4-NOT subunits confirmed it to be generally similar to the yeast complex in composition, with CNOT1 as a scaffold and interacting with several CNOT proteins (CNOT2, CNOT7, CNOT8, and CNOT9; Lau et al., 2009). However, several differences were observed between the yeast and human complexes. For instance, multiple deadenylases are observed in human cells with different binding properties, although both human and yeast CCR4-NOT complexes contain only one Ccr4 and one Caf1 subunit. Whilst CNOT3 was not observed to bind directly to CNOT1 in yeast two-hybrid experiments, but instead binds to CNOT2 and integrates into the complex via this interaction, this view has been superseded by recent structural evidence (discussed below). Finally, CNOT4 is a true ortholog of yeast Not4p but, unlike its yeast counterpart, it appears not to be stably integrated into the human complex although it can interact with the scaffold protein CNOT1. More recently, the Drosophila melanogaster CCR4-NOT complex was shown to share a similar composition to the human CCR4-NOT complex (Temme et al., 2010; Bawankar et al., 2013). D. melanogaster NOT4, a homolog of human CNOT4, is also not stably integrated into the complex. Two additional components, NOT10 and NOT11/C2orf29, were identified to dock onto the NOT1 N-terminal domain and are conserved in both the human and D. melanogaster complexes (Bawankar et al., 2013; Mauxion et al., 2013).

Increasing numbers of studies on the properties of the CCR4NOT complex has led to greater understanding about its threedimensional structure and its precise roles in the cell. In recent years, the availability of information on the structure and architecture of the CCR4-NOT complex has increased dramatically. In this review, we focus on the structure and architecture of the multi-subunit and multifunctional CCR4-NOT complex, particularly the components involved in deadenylation and mRNA degradation and the more recently characterized NOT module. We aim to summarize the current state of structure-function studies pertaining to the CCR4-NOT components.

\section{ARCHITECTURE OF THE CCR4-NOT COMPLEX}

To date, two studies have provided detailed information about the overall architecture of the CCR4-NOT complex. In the first, a low-resolution electron microscopy (EM) reconstruction of the 1.0 MDa yeast CCR4-NOT complex isolated using Not1 as bait reveals an L-shaped structure with two arms of similar length (180-190 A; Nasertorabi etal., 2011). The CCR4-NOT complex, including the subunits Not1-Not5, Ccr4, Caf1, Caf40, and Caf130, is natively heterogeneous and the authors were only able to obtain homogeneous L-shaped particles by chemical crosslinking of its components. Interestingly, using Caf1 as bait only yielded a Not1-Ccr4-Caf1 complex. The authors reported that relative arrangement of the arms varied between different reconstructions, indicating the whole assembly is flexible and therefore limiting resolution of the resulting EM map. Although positions of the subunits were not experimentally validated, prediction by the authors based on size consideration suggests that Not1Ccr4-Caf1 is located in the hinge region connecting the two arms (Figures 1A,B), where an accessible cavity could provide a platform for interaction with RNA or regulating factors. The NOT module, which has been shown to affect the stability and deadenylase activity of the complex, is predicted to lie in the bulkier arm of the complex adjacent to the deadenylase module (Figures 1A,B), thus rationalizing how crosstalk can occur between the two modules. The N-terminal region of Not1 (154-753) would then extend into the thinner arm of the complex where it could interact with other subunits, such as Caf40 and Caf130 in the yeast complex or CNOT10 and CNOT11 in the mammalian complex. This arrangement of subunits in the CCR4-NOT complex is in good agreement with a model constructed with currently available structures of the deadenylase and NOT modules (Figure 1). Tob/BTG proteins bind directly to Caf1, while other regulators could be recruited to either the extended $\mathrm{N}$-terminal HEAT-repeat platform or the C-terminal NOT module.

Bawankar et al. (2013) mapped the interactions of the individual subunits in the Drosophila melanogaster CCR4-NOT complex by co-immunoprecipitation and pull-down assays. The results not only confirmed the interactions that have been reported in various species, particularly the yeast and human complexes, they extended the interactions and provided possibly the most complete view of the CCR4-NOT complex to date. The results confirmed that NOT1 (homologous to CNOT1/Not1) is a scaffold protein for assembly of the complex, including the conserved NOT and deadenylase modules. The authors delineated three regions of NOT1 (CNOT1/Not1). The deadenylase module binds to the middle region via a MIF4G domain in NOT1 (CNOT1/Not1), with POP2 (CNOT7/Caf1) serving as an adaptor between NOT1 (CNOT1/Not1) and CCR4 (CNOT6L/Ccr4). A neighboring DUF3819 domain of unknown function in the middle region serves as the binding site for CAF40 (CNOT9/Caf40). The NOT module interacts with the C-terminal region of CNOT1/Not1. In their interaction map, NOT2 (CNOT2/Not2) interacts with NOT1 (CNOT1/Not1), whereas NOT3 (CNOT3/Not5) interacts with NOT2 (CNOT2/Not2) via their respective NOT boxes and has no direct interaction with NOT1 (CNOT1/Not1). Subsequent structural analysis has revised this view (see below). Two studies on the human and D. melanogaster complexes also showed that the N-terminal residues of CNOT1/Not1 interact with two proteins, CNOT10 (NOT10 in D. melanogaster) and CNOT11/C2orf29 (NOT11), which have no obvious orthologs in yeast (Mauxion et al., 2013; Bawankar et al., 2013). The functional significance of this CNOT10/CNOT11 module is unclear but, as it is not observed in yeast, it has been proposed to be an optional module or a functional homolog of the yeast Caf130 protein.

\section{THE CCR4-NOT DEADENYLASES}

The CCR4-NOT complex includes two distinct deadenylase enzymes belonging to different families (see Bartlam and 


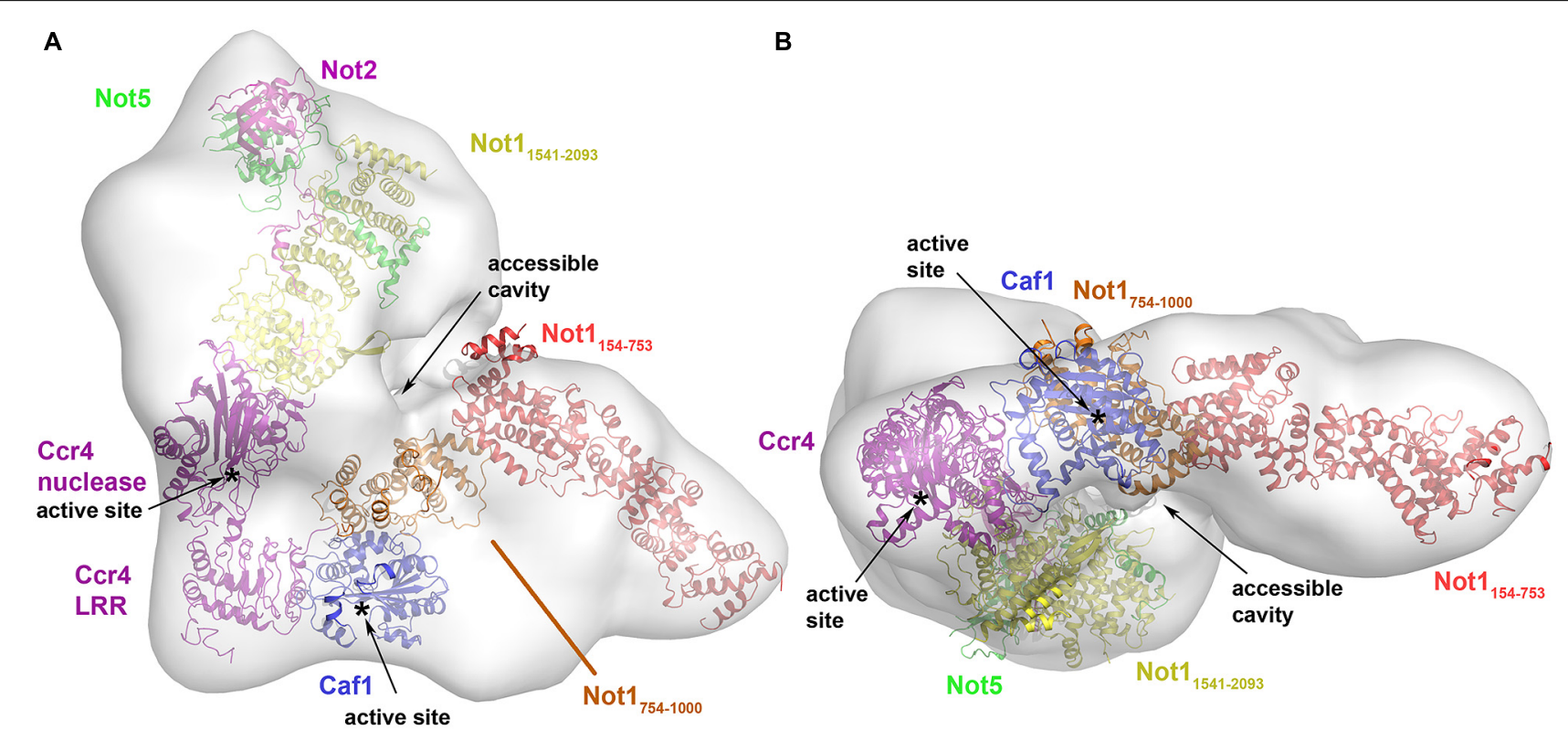

C

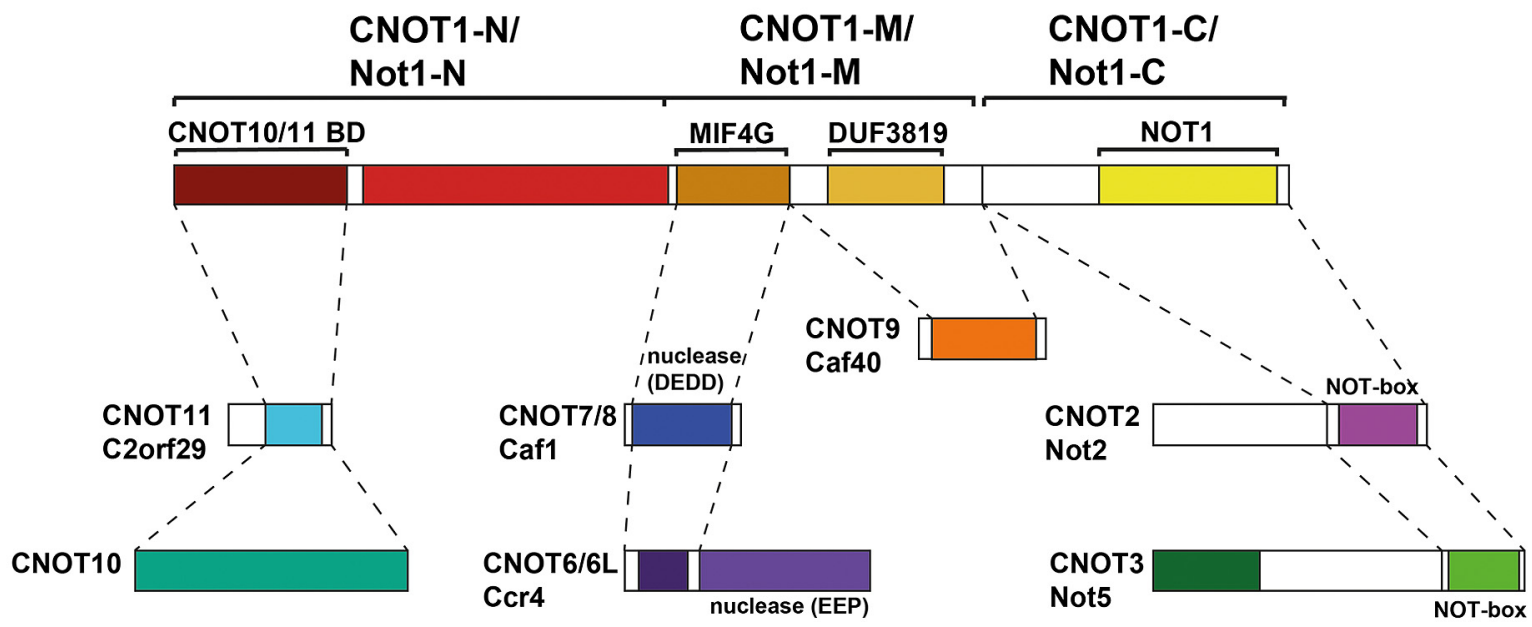

FIGURE 1 | Architecture of the CCR4-NOT complex. (A,B) Model of the CCR4-NOT complex using X-ray crystal structures docked into a negative-stain EM map of the yeast CCR4-NOT complex (EMDB ID: 1901; Nasertorabi etal., 2011). Crystal structures of CCR4-NOT subunits were docked into the EM map using the UCSF Chimera software based on size and shape considerations and guided by the prediction of Nasertorabi and colleagues. Structures used for docking are:
N-terminal domain of Not1 (PDB ID: 4B89); Not1-Ccr4-Caf1 (PDB ID: 4B8C): CNOT6L nuclease domain (PDB ID: 3NGQ); Not1-Not2-Not3 (PDB ID: 4BY6). The active sites of Ccr4 and Caf1 and the accessible cavity in the CCR4-NOT complex are labeled. (C) Map of the interactions in the CCR4-NOT complex (adapted from Bawankar etal., 2013). Subunits or domains are colored according to structures in (A,B) where available.
Yamamoto, 2010 for a review). The first are the DEDD-type nucleases, so named after the conserved catalytic Asp and Glu residues in three exonuclease motifs, including POP2, CAF1Z, poly(A)specific ribonuclease (PARN), and PAN2. The second are the EEP-type nucleases, or exonuclease-endonuclease-phosphatase, including CCR4, Nocturnin, ANGEL, and 2' phosphodiesterase ( $\left.2^{\prime} \mathrm{PDE}\right)$. The CCR4-NOT complex is characterized by the presence of one deadenylase enzyme from each family. The yeast CCR4-NOT complex encompasses two deadenylases, Ccr4 and Caf1 (Pop2), both of which are involved in mRNA degradation, although Caf1 is dispensable for the deadenylase activity of
Ccr4. The human CCR4-NOT complex is known to have two orthologs for each of the yeast deadenylases, Ccr4 and Caf1 (Bogdan et al., 1998; Albert et al., 2000; Dupressoir et al., 2001). The two human orthologs of yeast Ccr4 are CNOT6 (CCR4a) and CNOT6L (CCR4), which evidently have different functions as CNOT6L is involved in cell proliferation in mouse 3T3 fibroblasts but CNOT6 is not (Morita et al., 2007). This phenomenon appears to be celltype specific, as both CNOT6 and CNOT6L are required for cell viability in MCF7 cells (Mittal et al., 2011). The two orthologs of Caf1 are CNOT7 (CAF1) and CNOT8 (POP2/CALIF), which share high sequence similarity, have partially overlapping function 
and are both important for cell proliferation (Aslam et al., 2009). Lau et al. (2009) identified distinct human CCR4-NOT complexes with either CNOT7 or CNOT8, implying that they compete for the same binding site on CNOT1. In the same study, CNOT6 and CNOT6L are mutually exclusive in CCR4-NOT complexes (Lau et al., 2009). Structures are presently available for representatives of each family and will be discussed in more detail below.

\section{DEDD-TYPE DEADENYLASES}

The crystal structure of Cafl was first determined from $S$. cerevisiae in 2003 (Thore et al., 2003), and then from Schizosaccharomyces pombe in 2007 (Jonstrup etal., 2007; Andersen etal., 2009; Figure 2). Cafl adopts a kidney-shaped structure with $13 \alpha$ helices and $6 \beta$-strands (Figure 2A). Comparison with related structures placed Caf1 as a member of the DEDD-type nucleases with the common fold of the DnaQ subgroup. DEDD-type nucleases are so named because they feature an Asp-Glu-AspAsp sequence motif in their active site. Despite low sequence similarity, Cafl shares structural similarity with the $\epsilon$-subunit of DNA polymerase III and the exonuclease domain of DNA polymerase I.

The structure of S. pombe Caf1 provided insight into the selectivity and activity of this class of deadenylase (Jonstrup et al., 2007). Two divalent metal ions (sites A and B) located in the active site were found to be essential for activity (Figures 2A,B). Interestingly, although $\mathrm{Mg}^{2+}$ is found in higher concentrations inside cells, the A site of $S$. pombe Caf1 was found to favor $\mathrm{Zn}^{2+}$ and the $\mathrm{B}$ site exhibited a preference for $\mathrm{Mn}^{2+}$, despite a large 100-fold excess of $\mathrm{Mg}^{2+}$ in the cell. The composition of the two ions in the active site was also found to have an effect on the kinetics of deadenylation. In the presence of $\mathrm{Mg}^{2+}, \mathrm{Mn}^{2+}$, and $\mathrm{Zn}^{2+}$ ions, deadenylation was slow and unspecific, and only $\sim 25 \%$ of RNA substrates were completely deadenylated after $160 \mathrm{~min}$. In the absence of $\mathrm{Zn}^{2+}$, however, Caf1 was observed to quickly and specifically degrade the complete poly(A) tail of the RNA substrate, suggesting that variations in cellular $\mathrm{Zn}^{2+}$ levels might provide a means to regulate the overall rate of mRNA turnover. The S. cerevisiae Caf1 revealed that the consensus DEDD nuclease motif is substituted by an SEDQ motif and so it lacks several essential highly conserved catalytic residues, implying it may have lost its catalytic activity and that its role might be architectural, such as to recruit Ccr4 into the CCR4-NOT complex. Caf1 was shown to function as an active exonuclease in vitro on monotonous RNA sequences, with a slight preference for poly(A) RNA over poly $(\mathrm{U})$ and poly $(\mathrm{C})$. This lack of absolute specificity suggests that Caf1 may not bind RNA substrates in a specific manner, but may involve a substantial contribution of van der Waals interactions.

The structure of human CNOT7, an ortholog of yeast Caf1, was reported in complex with the antiproliferative protein Tob (Figure 2C; Horiuchi et al., 2009). Unsurprisingly, CNOT7 shares high structural similarity with Caf1 from S. pombe and S. cerevisiae, as well as to a number of DEDD-type nucleases including human PARN. The CNOT7 structure was found to adopt the core catalytic domain of the RNase D superfamily, characterized by the DEDD sequence motif. These conserved acidic amino acids are responsible for metal ion binding. As with the $S$. pombe Caf1 structural study, Horiuchi et al. (2009) examined the nuclease activity of CNOT7 in the presence of the metal ions $\mathrm{Mg}^{2+}, \mathrm{Ca}^{2+}, \mathrm{Mn}^{2+}$, and $\mathrm{Co}^{2+}$. No activity was observed in the presence of $\mathrm{Ca}^{2+}$, and CNOT7 exhibited significantly higher activity for RNA substrates over DNA substrates. Highest RNase activity was observed in the presence of $\mathrm{Mn}^{2+}$, suggesting that $\mathrm{Mn}^{2+}$ is required for full activity of CNOT7 (Horiuchi et al., 2009). A recent structure of the human CNOT1-CNOT7 complex by Petit etal. (2012) showed two $\mathrm{Mg}^{2+}$ ions in the $\mathrm{A}$ and $\mathrm{B}$ sites of CNOT7, although the two metals are unusually close at $3.9 \mathrm{~A}$ due to additional coordination of the $\mathrm{A}$ site $\mathrm{Mg}^{2+}$ ion by Glu278, as opposed to 4.6 A between ions in S. pombe Caf1.

Tob, a regulator that promotes mRNA degradation, is not present in yeast but accumulating evidence indicates that the Tob/BTG family members are common binding partners of CNOT7 in the human CCR4-NOT complex (Mauxion et al., 2009; Winkler, 2010). The interaction of Tob with CNOT7 is

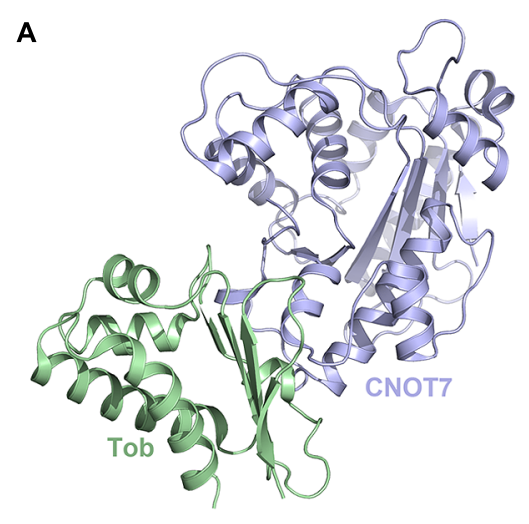

FIGURE 2 |The DEDD-type deadenylases. (A) Crystal structure of the human CNOT7-Tob complex (PDB ID: 2D5R). (B) Crystal structure of $S$. pombe Caf1 (PDB ID: 3G0Z). (C) Enlarged view of the $S$. pombe Caf1 active
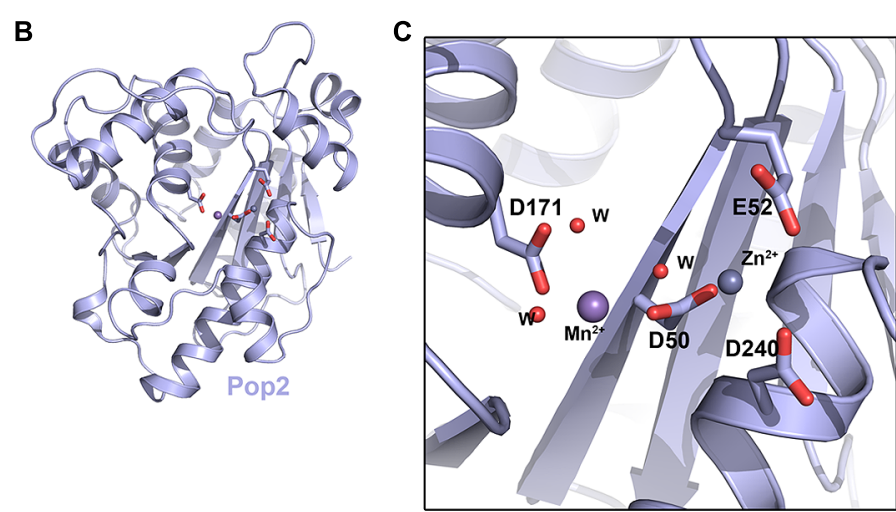

site showing the DEDD motif in stick representation. $\mathrm{Mn}^{2+}$ and $\mathrm{Zn}^{2+}$ ions in the active site are shown as spheres and waters are shown as small red spheres. 
largely hydrophobic and mediated by Box A and Box B regions in the N-terminal BTG domain that are conserved throughout the Btg/TOB family, as observed in structures of human BTG2 and mouse TIS21 (Yang et al., 2008). The C-terminal region of Tob features a conserved PAM2 motif required for interaction with the C-terminal domain of PABP, suggesting that Tob serves as an adaptor protein for the interaction between the CCR4-NOT complex and PABP (Ezzeddine et al., 2007; Funakoshi et al., 2007). This is in contrast to the PAN2-PAN3 complex, which interacts directly with PABP via a PAM2 motif in PAN3 (Siddiqui et al., 2007). Funakoshi et al. (2007) proposed a hypothesis in which recruitment of a PABP-mRNA complex to the CCR4-NOT complex via the Tob-CNOT7 interaction would cause translation to be interrupted by rapid degradation of the poly(A) tail, presumably by CNOT6 or CNOT6L, followed by repression of cell growth.

\section{EEP-TYPE DEADENYLASES}

Ccr4 was identified to be the main deadenylase in yeast by the Denis (Malvar etal., 1992; Draper etal., 1994; Chen et al., 2002) and Parker (Tucker etal., 2001) labs. To date, the only structure of an intact Ccr4 nuclease domain is that of human CNOT6L (Wang etal., 2010), although a partial structure of the yeast Ccr4 nuclease domain has been reported as part of the Not1-Ccr4-Caf1 ternary complex (Basquin et al., 2012). The heart-shaped fold of CNOT6L features a two-layered $\alpha-$ $\beta$ sandwich: two interior $\beta$-sheets are sandwiched between outer layers of $\alpha$-helices (Figure 3A). A prominent active-site pocket at the top of the molecule features two bound $\mathrm{Mg}^{2+}$ ions (Figures 3A,B). Within the active site, the indispensable residue Glu240 coordinates the first, stable ion; mutation of Glu240 or absence of $\mathrm{Mg}^{2+}$ abolished enzymatic activity, showing that $\mathrm{Mg}^{2+}$ is a necessary cofactor (Figure 3B). Asp410, Asn412, and His529 coordinate the second, weakly bound magnesium ion. Subsequent experiments confirmed that the deadenylase activity of CNOT6L is $\mathrm{Mg}^{2+}$-dependent (Wang et al., 2010).

Deadenylase activity assays performed on $\operatorname{poly}(A), \operatorname{poly}(G)$, poly $(\mathrm{C})$, and poly $(\mathrm{U})$ RNA substrates indicated that CNOT6L has strict substrate specificity for poly(A) RNA substrates, while similar experiments comparing activity for poly(A) RNA and DNA substrates revealed only trace activity for poly(A) DNA (Wang et al., 2010). Structural studies of CNOT6L with riboadenine- $5^{\prime}$-monophosphate (rAMP) and a $5 \mathrm{~A}$ stretch of poly $(\mathrm{A})$ DNA were employed to probe the structural basis for this strict substrate specificity. In the poly(A) DNA structure, a $3 \mathrm{~A}$ trinucleotide substrate was observed in the binding site with the scissile phosphate of $\mathrm{A}_{2}$ fixed by three bonds: two coordinated bonds with the $\mathrm{Mg}^{2+}$ ions and a third between Asn412 and the dissociative $\mathrm{O}$ atom of the phosphate moiety (Figure 3C). The adenine base of $\mathrm{A}_{2}$ stacks between the phenyl ring of Phe 484 and the ring of Pro365, while a further bond is formed between the $6^{\prime}-\mathrm{NH} 2$ group of the adenine base and Asn412. A near-identical conformation of rAMP indicates the importance of Pro365, Asn412, and Phe484 in substrate recognition (Figure 3C): mutating the latter two abolished activity, while mutating Pro365 reduced activity. The purine base $G$, which shares the greatest similarity with $A$, has a carbonyl oxygen in the $6^{\prime}$ position that would clash with Asn412, while the pyrimidine base and $4^{\prime}-\mathrm{NH}_{2}$ or carbonyl group of $\mathrm{C}$ or $\mathrm{U}$ bases could not be accommodated within the recognition pocket. Nucleotides $A_{1}$ and $A_{3}$ in the poly(A) DNA complex do not form specific interactions with CNOT6L.

\section{THE DEADENYLASE MODULE}

The first structural view of a complete CCR4-NOT nuclease or deadenylase module was reported in 2012 for the yeast complex (Basquin etal., 2012; Figure 4). The ternary complex structure is formed between the N-terminal arm of Not1, Ccr4, and
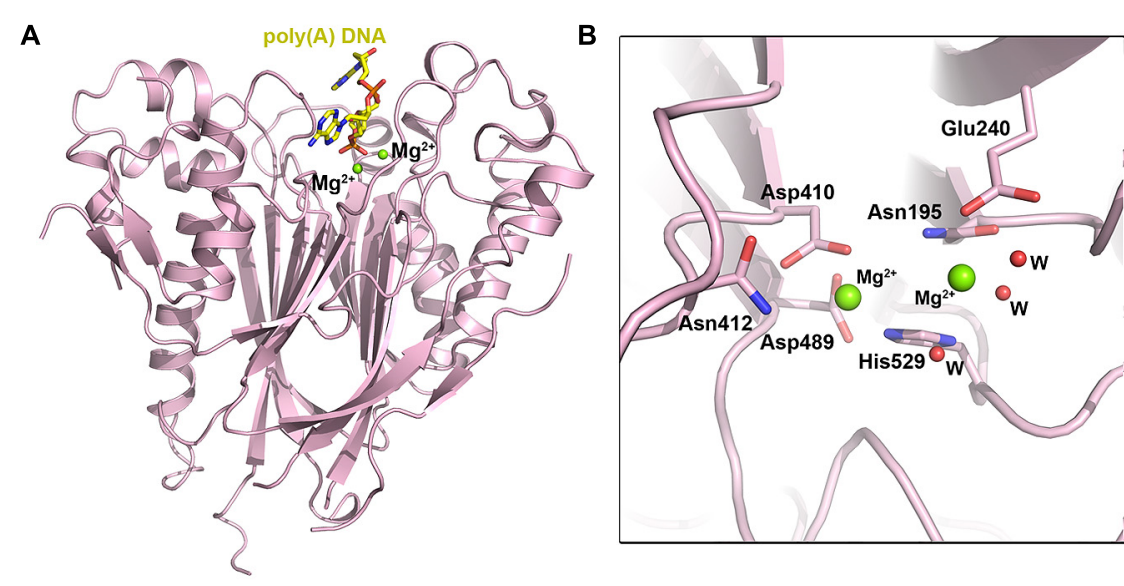

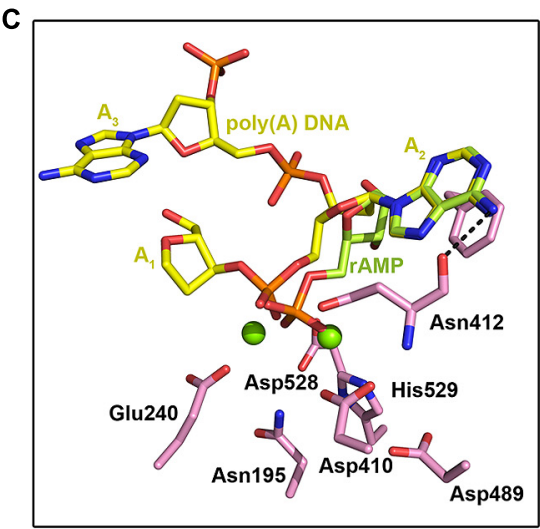

FIGURE 3 |The EEP-type deadenylases. (A) Crystal structure of the human CCR4-NOT nuclease domain in complex with poly(A) DNA (PDB ID: 3NGO). The DNA ligand is shown in yellow stick representation and $\mathrm{Mg}^{2+}$ ions are shown as spheres. (B) Enlarged view of the human CNOT6L active site showing active site residues in stick representation. $\mathrm{Mg}^{2+}$ ions are shown as green spheres and waters are shown as small red spheres. (C) Substrate binding by human CNOT6L, showing poly(A) DNA (yellow stick representation) and rAMP (pale green stick representation) in the substrate binding pocket. CNOT6L active site residues are shown in pink and $\mathrm{Mg}^{2+}$ ions are shown as green spheres. 


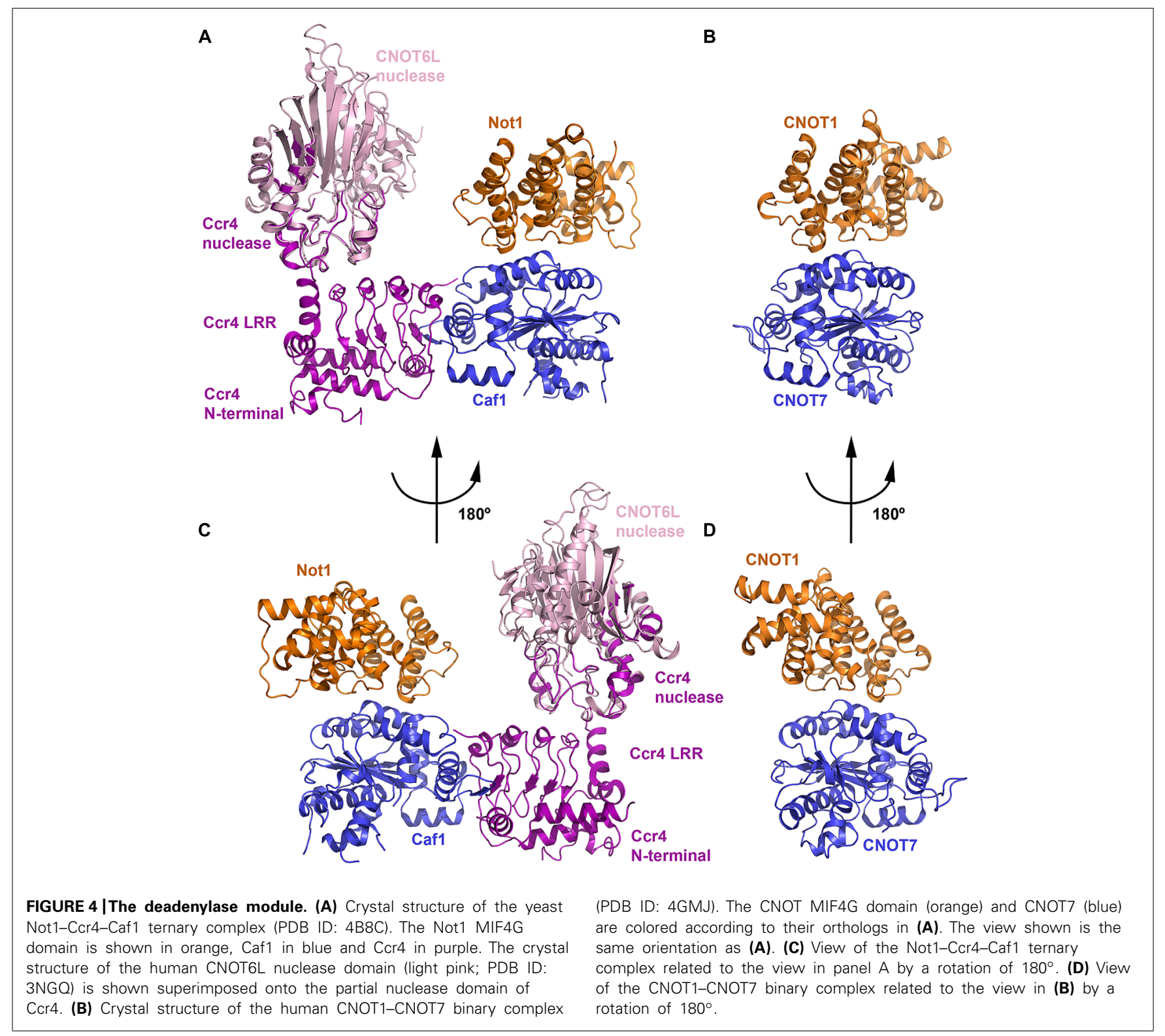

Caf1. The N-terminal arm of Not1 (residues 754-1000) forms a structure of five HEAT repeats related to the MIF4G (middle domain of initiation factor $4 \mathrm{G}$ ) fold. Each HEAT motif consists of two antiparallel helices, termed A and B helices, and successive HEAT motifs pack together in an almost parallel manner. The CNOT1 MIF4G domain features a concave surface with a large patch of conserved residues contributed by the B helices of HEAT motifs 1-5. Another small patch of conserved residues is present on the opposite surface located in the interrepeat loops between HEAT3-4 and HEAT4-5. Although no structure exists for the complete vertebrate CCR4-NOT deadenylase module, Petit et al. (2012) reported a structure of the binary complex between human CNOT1-MIF4G (residues 1093-1317) and CNOT7.

In both human and yeast structures, CNOT7/Caf1 is recognized by and interacts with the small conserved hydrophobic patch in the CNOT1/Not1 MIF4G domain formed by the HEAT3-4 and HEAT4-5 inter-repeat loops (Figure 4). CNOT7/Caf1 binds CNOT1/Not1 at the opposite side to the active-site pocket, such that the active site is solvent exposed in the ternary complex. Only $8.4 \%$ of total accessible solvent area is buried in the Not1-Caf1 interface, leaving a large conserved patch of the Notl concave surface accessible and exposed to solvent. Other regulatory factors may be recruited to the CCR4-NOT complex via this region. For instance, tristetraprolin (TTP) has been shown to bind to this MIF4G domain of CNOT1 (see below). Residues involved in the interaction between CNOT7/Caf1 and the CNOT1/Not1 MIF4G domain are primarily hydrophobic and are evolutionarily conserved.

Ccr4 interacts with Caf1 via its LRR (leucine rich repeat) domain (Figures 4A,C), which contains five repeats assembled into a crescent-shaped structure, but makes no direct interaction 
with Not1 (Basquin et al., 2012). The N-terminal region of Ccr4 interacts intermolecularly with the LRR domain, forming a single structural unit, while the 70 amino acid linker between the $\mathrm{N}$-terminal region and LRR domain is disordered. In vertebrates, Ccr4 orthologs lack the $\mathrm{N}$-terminal region and the concave surface of the LRR domain is polar. Half of the nuclease domain in the crystal structure is disordered, and the helices in the resolved half interact with the LRR domain. The interface between LRR and nuclease domains buries a surface area of about $400 \AA^{2}$ and involves both hydrophobic and polar contacts. Superimposing the structure of the intact human CNOT6L nuclease domain (Wang et al., 2010) onto the partial Ccr4 nuclease domain shows that the active site does not contact Not1 and remains accessible.

Based on structural analysis, Basquin et al. (2012) studied the effects of mutants designed to disrupt the Not1-Caf1 and Caf1$\mathrm{Ccr} 4$ interactions in vivo. While Ccr4-Caf1 are active deadenylases in the absence of Not1 in vitro, the in vivo results confirmed that incorporation of Caf1 and Ccr4 into the CCR4-NOT complex is essential for efficient deadenylation in vivo. Disrupting the association of Cafl with Not1 resulted in a poor growth phenotype at high temperature, and mRNA decay intermediates accumulated when the ternary complex was disrupted. This is in good agreement with the observation by Petit et al. (2012) that a D. melanogaster CAF1 mutant that does not interact with NOT1 has a dominant-negative effect on mRNA degradation, inhibiting it by possibly sequestering CCR 4 and preventing its incorporation into the endogenous CCR4-NOT complex. An intact complex may therefore be required for full efficient deadenylase activity, as the complex might target specific mRNA substrates or additional CCR4-NOT subunits may contribute to the activity. For instance, depleting CNOT2 is known to disrupt stability of the CCR4-NOT complex and inhibit its deadenylase activity, leading to apoptosis (Ito et al., 2011a).

\section{THE NOT MODULE}

The second major module in the CCR4-NOT complex is formed by CNOT1, CNOT2, and CNOT3 in the human complex, and by Not1, Not2, and either Not3 or Not5 in the yeast complex. Both CNOT2 and CNOT3 in the human complex possess a NOT-box motif that has been shown to mediate their interaction (Zwartjes et al., 2004; Bawankar et al., 2013). In the yeast complex, Not2 is an ortholog of CNOT2, while Not3 and Not5 are orthologs of CNOT3 and thought to be paralogous proteins resulting from a gene duplication event (Collart et al., 2013). Sharing similar Ntermini but distinct C-termini, Not5 is critically important for yeast vegetative growth whereas Not3 has only very mild phenotypes (Oberholzer and Collart, 1998). Whilst Not3 is predicted to have a similar fold and dimerization interface as Not 2 and Not5, it does not bind to a Not2- $\Delta \mathrm{N}$ mutant in contrast to Not5 and a subset of the dimerization interface diverges between the two paralogs (Bhaskar et al., 2013). The precise function of the NOT module remains unclear, but it has been linked to regulation of the CCR4-NOT complex stability and activity, and to recruitment of specific mRNAs (Wahle and Winkler, 2013). It has also been linked to poly(A) tail shortening, but its overall involvement in mRNA degradation is unclear. The NOT module also has distinct functions from the deadenylase module (Bai et al., 1999): for instance, it has been linked to transcription machinery [see (Collart and Panasenko, 2012; Miller and Reese, 2012) for reviews] and cytoplasmic mRNA decay pathways (Muhlrad and Parker, 2005; Temme et al., 2010).

Recent three-dimensional structures of the human CNOT1CNOT2-CNOT3 (Boland et al., 2013) and yeast Not1-Not2-Not5 (Bhaskar etal., 2013) complexes have revealed the structure and assembly of the NOT module (Figure 5). Each structure shares a similar architecture in which CNOT2/Not2 and CNOT3/Not5 form a symmetric heterodimer via their NOTbox motifs, referred to as the symmetric lobe (Figures 5A-D). Extended N-terminal sequences, termed connector sequences (CSs) by Boland etal. (2013), extend the heterodimer interface by wrapping around the NOT-box of their neighbor. As the CSs of CNOT2/Not2 and CNOT3/Not5 differ in length and sequence, it is proposed that this structural divergence favors heterodimerization over homodimerization. Boland et al. (2013) observed that free CNOT2 and CNOT3 constructs containing both NOT-box and CSs tend to aggregate and precipitate in isolation, indicating that CSs are not favorable for homodimerization.

The heterodimer between CNOT2/Not2 and CNOT3/Not5 is tightly anchored to CNOT1/Not1 via unstructured, extended peptide regions, referred to as the asymmetric lobe (Figure 5). The C-terminal region of CNOT1/Not1 that forms the NOT module consists of 10 HEAT repeats, each characterized by a helix A-turn-helix B motif. The 10 HEAT repeats in the CNOT1/Not1 C-terminal region can be further divided into two units: HEAT motifs 1-6, and HEAT motifs 7-10 (Figure 5). The short CNOT3/Not5 anchor wraps around HEAT motifs $1-5$ and interacts via hydrophobic and polar residues. The long CNOT2/Not2 anchor region zigzags across the surface of CNOT1/Not1, starting at HEAT motifs 9-10 and interacting with HEAT motifs 4-6. Notably, a conserved hydrophobic pocket in yeast Not1 formed by Leu2027, Leu2031, Phe2064, and Ile2071 recognizes Leu9 of Not2 (Bhaskar et al., 2013). An L9P mutant of Not2 results in loss of integrity of the S. cerevisiae CCR4-NOT complex, highlighting its functional importance in vivo (Russell et al., 2002).

The NOT-boxes of CNOT2/Not2 and CNOT3/Not5 are contained within their globular C-terminal domains. The NOT-box structure consists of three $\mathrm{N}$-terminal $\alpha$-helices and a C-terminal $\beta$-sheet formed by four (yeast) or five (human) $\beta$-strands, with considerable bending of strands $\beta 3 / \beta 4$ (yeast) or $\beta 4 / \beta 5$ (human). The NOT-boxes are most similar to Sm domains, but differ in that they lack signature Sm sequence motifs and lack an additional $\beta$-strand that mediates Sm-Sm dimerization. The NOT-box domains of CNOT2/Not2 and CNOT3/Not5 mediate dimerization via their $\mathrm{N}$-terminal helices, resulting in a highly symmetrical heterodimer that adopts the same arrangement as a CNOT3 homodimer (Figure 5E; Boland et al., 2013) albeit with greater complementarity. A CNOT2 homodimer structure features domain swapping via the $\mathrm{N}$-terminal helices that results in a distinct mode of dimerization from the CNOT3 homodimer and the CNOT2/CNOT3 heterodimer (Figures 5E-G). The NOT-box of CNOT3/Not5 is highly similar to that of CNOT2/Not2 and can be superimposed with an r.m.s.d. of $<1.3 \AA$ (Figure $5 \mathbf{H}$ ). 
A
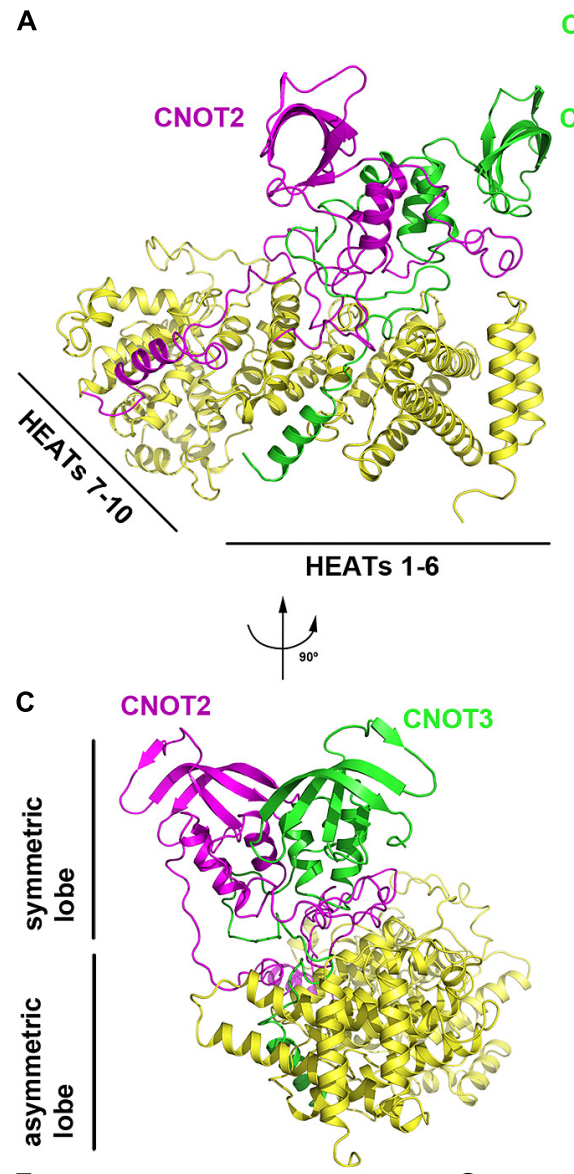

E
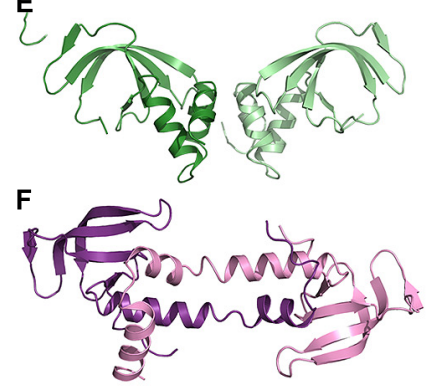

G
CNOT3 B

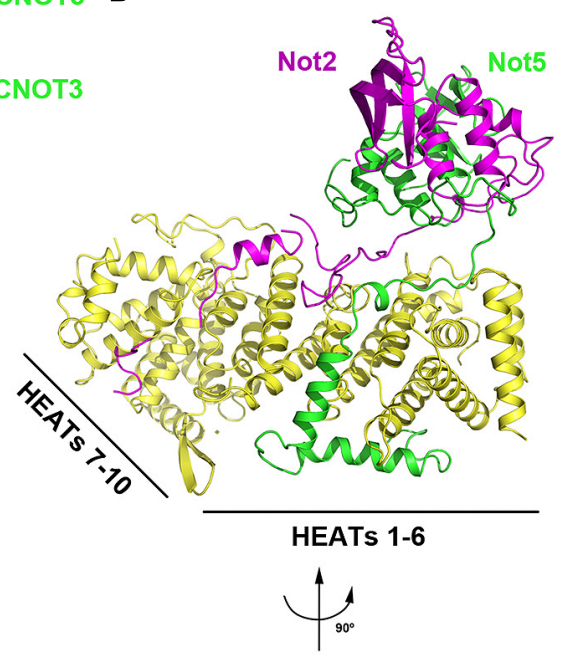

D

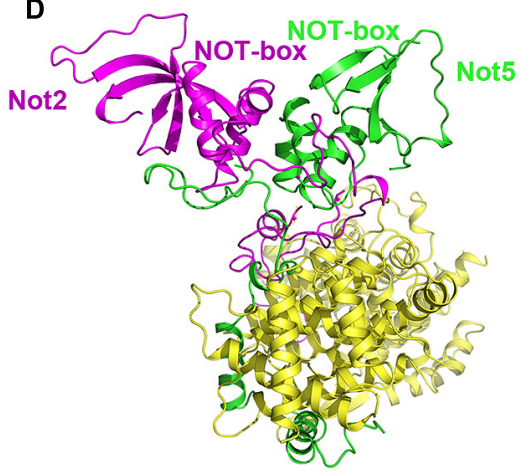

H

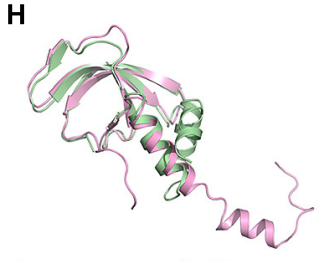

FIGURE 5 | The NOT module. (A) Crystal structure of the human CNOT1-CNOT2-CNOT3 ternary complex (PDB ID: 4COD). CNOT1 is shown in yellow, CNOT2 in magenta and CNOT3 in green. (B) Crystal structure of the yeast Not1-Not2-Not5 ternary complex (PDB ID: 4BY6). Not1 is shown in yellow, Not2 in magenta and Not5 in green. The complex is shown in the same orientation as (A). (C) View of the human CNOT1-CNOT2-CNOT3 complex related by $90^{\circ}$ to the orientation in (A). (D) View of the human
Not1-Not2-Not3 complex related by $90^{\circ}$ to the orientation in (B). (E) Crystal structure of the human CNOT3 NOT-box homodimer (PDB ID: 4COG). (F) Crystal structure of the human CNOT2 NOT-box homodimer (PDB ID: 4COF). (G) The CNOT2-CNOT3 heterodimer (PDB ID: 4COD) shown in the same orientation as panel E. CNOT2 is shown in purple and CNOT3 is shown in green. (H) Superposition of the CNOT2 (PDB ID: 4COF; pink) and CNOT3 (PDB ID: 4COG; green) NOT-box structures.
Bhaskar etal. (2013) further revealed that the ternary Not1Not2-Not5 complex forms a surface that binds poly(U) RNA in vitro, with mass spectrometry analysis identifying a nucleotidebinding site in Not5 centered around Cys546, different from the canonical nucleotide binding site of Sm folds. A U15 RNA substrate did not bind to Not1 or Not2-Not5 in isolation, suggesting that the three subunits cooperate to bind nucleotides, and the structure reveals a surface patch adjacent to Cys546 at the interface between Not1, Not2, and Not5. RNase protection assays used to estimate the length of the RNA-binding path on the complex indicated the accumulation of 11-15 nucleotide fragments, spanning a distance of 45-60 A. Mouse CNOT3 has previously been shown to regulate deadenylation of specific mRNAs via recruitment of their $3^{\prime}$ UTRs, which contain U-rich sequences (Morita et al., 2011). In yeast, Edc1 mRNA decay occurs via a deadenylation-independent pathway 
involving CCR4-NOT components and a poly-U tract in the $3^{\prime}$ UTR.

Based on their structural analysis, Boland et al. (2013) examined the integrity of the NOT module and its effects on mRNA degradation. In D. melanogaster, wild-type Not1 had no effect on mRNA half-life whereas a $\triangle N$-SD mutant resulted in a two-fold increase in mRNA half-life. The $\Delta \mathrm{N}$-SD Notl mutant was still able to bind Caf1, leading the authors to suggest that the catalytic module is sequestered into inactive or defective complexes that are no longer recruited to the mRNA reporter. Depletion of individual Not1, Not2, and Not3 subunits co-depleted the other two subunits, demonstrating strict control of respective protein ratios in the cell. Not1- and Not3-depleted cells also resulted in a ten-fold increase of hsp70 mRNA half-life relative to control cells. Not1 and Not3 mutants were also unable to restore mRNA degradation in Not1- and Not3-depleted cells in complementation assays. Taken together, they showed that an intact NOT module is essential for activity and recruitment of the CCR4-NOT complex and therefore for mRNA degradation.

\section{OTHER STRUCTURES}

In addition to the deadenylase and NOT modules, the CCR4-NOT complex includes several other subunits that are either peripheral to the core complex or species specific. The CNOT9/Rcd1 subunit (also known as RQCD1 or Caf40) has been shown to interact with CNOT1/Not1 via a central DUF3819 domain of unknown function (Figure 1; Bawankar et al., 2013). The crystal structure of CNOT9 has been determined and reveals an Armadillo repeat formed by a bundle of $18 \alpha$-helices arranged in a clockwork spiral (Figures 6A,B; Garces et al., 2007). The Armadillo motif is found in many diverse proteins with unrelated functions and, when folded together into a superhelix, provides a versatile platform for protein-protein interactions. CNOT9 was previously shown to interact with $\operatorname{poly}(\mathrm{G})$, poly $(\mathrm{C})$, and $\operatorname{poly}(\mathrm{T})$ oligonucleotides, but was reported to have little or no detectable affinity for poly(A) sequences (Garces et al., 2007). The authors observed a positively charged cleft on the surface of CNOT9 and identified Arg227 in the cleft as important for nucleotide binding. The functional significance of this oligonucleotide binding remains unclear. However, as CNOT9 appears to be located in close proximity to the deadenylase module in the CCR4-NOT complex (Nasertorabi et al., 2011; Bawankar et al., 2013), it may contribute in some way to the deadenylase activity.

Tristetraprolin (TTP) is an RNA-binding protein that interacts with the CCR4-NOT complex to post-transcriptionally repress gene expression (Sandler et al., 2011). It achieves this by interacting with AU-rich elements (AREs) in $3^{\prime}$ untranslated regions of target mRNAs and subsequently engenders their deadenylation and decay. Fabian et al. (2013) identified an evolutionarily conserved C-terminal motif in human TTP that binds to the central region of CNOT1. A subsequent crystal structure reveals that a TTP peptide from residues $312-326$ binds into a conserved hydrophobic groove between helices $\alpha 1$ and $\alpha 3$ of the CNOT1 MIF4G domain from residues 820-999 (Figures 6C,D). This is in good agreement with the finding by Sandler et al. (2011) that the CNOT1 region from amino acids 700-997 is important for the interaction with TTP. The central portion of the peptide forms a short two-turn amphipathic $\alpha$-helix that binds into the groove, while the termini of the peptide forms a network of electrostatic interactions with surrounding negatively charged residues in CNOT1. An in vitro assay for TTPmediated deadenylation showed that a mutant of TTP that could not bind to CNOT1 displayed severely impaired deadenylation, demonstrating that the TTP-CNOT1 interaction is required for TTP-mediated deadenylation in vitro. Similarly, TTP was found to require its C-terminal CNOT1-interacting motif for efficient decay of a target mRNA in vivo. The miRNA-associated protein GW182, which also binds to the CCR4-NOT complex via two CIMs, is believed to bind to different regions of CNOT1, as the TTP and GW182 CIMs share no homology (Fabian et al., 2011, 2013).

One key difference between yeast and vertebrate CCR4-NOT complexes is the CNOT4/Not4 protein, which has been shown to function as an E3 ubiquitin ligase. In yeast, Not4 is a stable member of the CCR4-NOT complex and interacts with Not1 via its C-terminus (Albert et al., 2002; Panasenko and Collart, 2011). In human cells, the homologous CNOT4 does not bind stably to CNOT1 and is only recruited to the CCR4-NOT complex under certain unspecified conditions (Lau et al., 2009), indicating that some functions of CNOT4 may be performed outside the complex. However, the Not4 function is conserved as human CNOT4 can partially compensate for the absence of the yeast protein (Albert etal., 2000). The yeast and human proteins share similar $\mathrm{N}$-terminal regions (44\% sequence identity over the first 230 amino acids of CNOT4) but divergent C-terminal regions, possibly reflecting their different associations with the CCR4-NOT complex (Figure 6E). The NMR structure of the CNOT4 RING domain has been determined and a structural model of the CNOT4 RING domain has been reported in complex with its E2 conjugating enzyme partner UbcH5B (Hanzawa et al., 2001; Dominguez et al., 2004), revealing the structural basis for E2/E3 specificity (Figure 6E). A solution structure has also been determined for the CNOT4 RNA-recognition motif (RRM, PDB ID: 2CPI; unpublished, Figure 6E). No structures are available for the C-terminal of CNOT4 or Not4, which has no identifiable domains. Known substrates of Not4 include the nascent polypeptide-associated Egd complex, a ribosomal chaperone, the ribosomal protein Rps7a, and the demethylase Jhd2. More recently, Not 4 has been shown to play a role in protein quality control via its involvement in proteasome assembly (Panasenko and Collart, 2011), and this role is distinct from the CCR4-NOT deadenylase activity (Halter et al., 2014).

\section{PERSPECTIVES}

Significant progress has been made in recent years towards understanding the three-dimensional structure and assembly of the CCR4-NOT complex. The interactions of the subunits in the core CCR4-NOT complex have been mapped and structures are now available for both the conserved deadenylase and NOT modules. Combining this wealth of structures at the atomic level with a low resolution negative staining EM map of the yeast CCR4-NOT complex facilitates modeling the CCR4-NOT complex (Figure 1), thus providing a more complete view of 
A

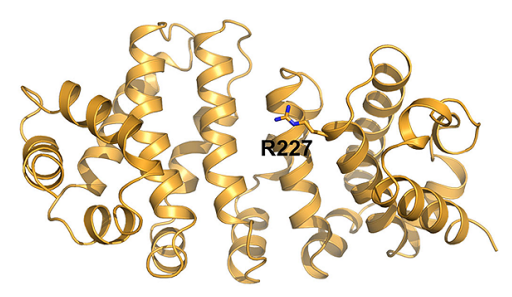

C

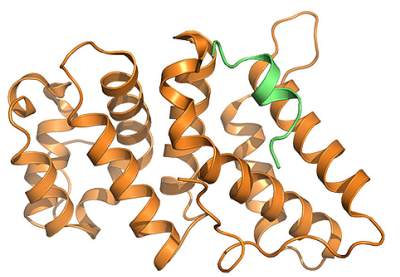

B

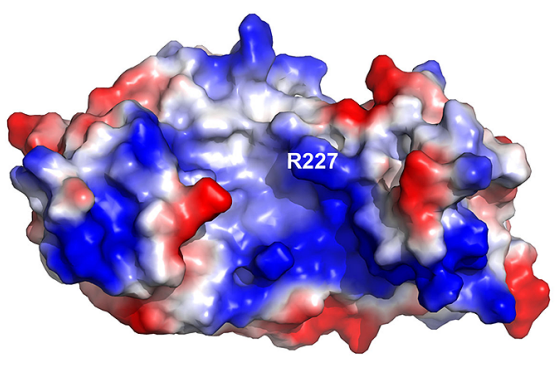

D

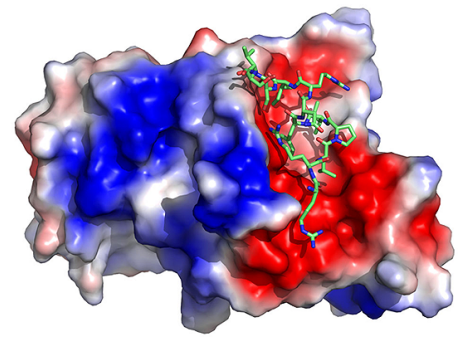

\section{E}
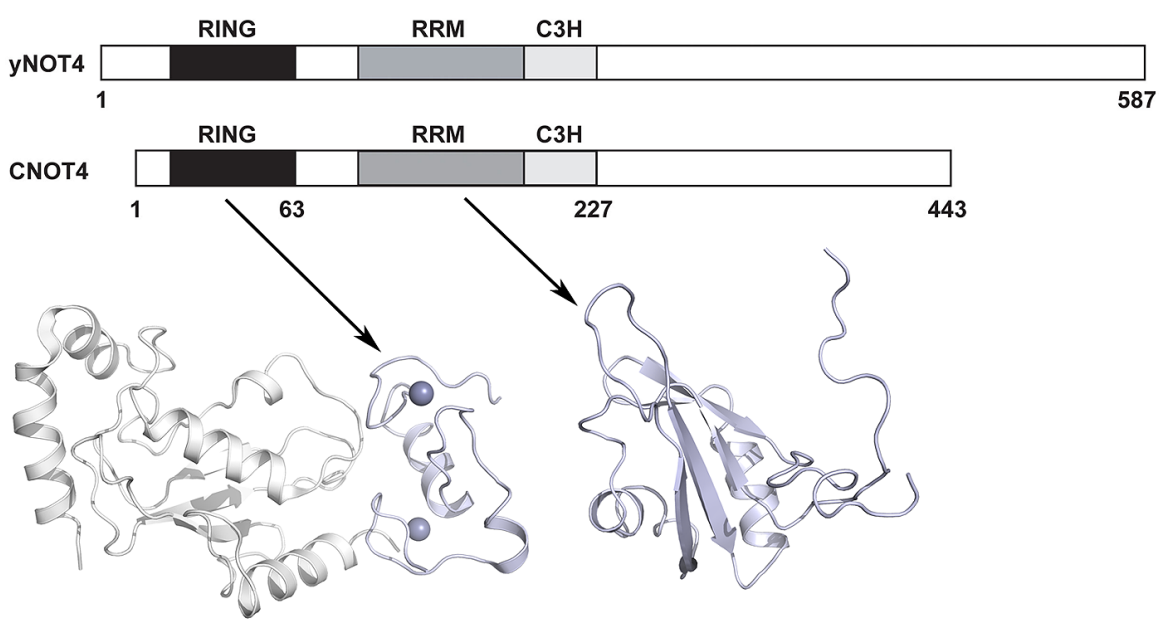

FIGURE 6 | Other CCR4-NOT protein structures. (A) Crystal structure of human CNOT9 (PDB ID: 2FV2). The amino acid Arg227 implicated in nucleic acid binding is shown in stick representation. (B) Electrostatic surface representation of human CNOT9 showing the position of Arg227 in a region of positive charge. (C) Crystal structure of the human CNOT1 MIF4G domain in complex with a TTP peptide (PDB ID: 4J8S). The CNOT1 MIF4G domain is colored orange and the TTP peptide is colored green. (D) Electrostatic surface representation of the CNOT1 MIF4G domain showing the binding surface for the TTP peptide, shown in green stick representation. (E) The domain organization of yeast Not4 and human CNOT4. Shown below are the structural model of the human CNOT4 RING-UbcH5b complex (left; PDB ID: 1UR6) and the solution structure of the RRM (right, PDB ID: 2CPI) domain. The CNOT4 RING and RRM domains are shown in pale blue, and UbcH5b is shown in white. the complex. However, it should be stressed that such models are only a prediction and further structure-function studies are needed to elucidate the functional roles of the deadenylase and NOT modules and to study the mechanism of crosstalk between them.

Another important question to be addressed is why the deadenylase module contains two deadenylase enzymes, Ccr4 and Cafl. It is unclear how these enzymes cooperate and whether they play specialized or redundant roles (Winkler and Balacco, 2013). Both Ccr4 and Caf1 have a preference for poly(A) RNA substrates, but $S$. cerevisiae Caf1 has a SEDQ motif in place of the DEDD motif in its catalytic site and may not be active in vivo, serving only a structural role in recruiting Ccr4 to the complex (Basquin et al., 2012). In human cells, it is further unclear why two paralogs exist for Ccr4 (CNOT6, CNOT6L) and for Caf1 (CNOT7, CNOT8).

Whilst the structure and assembly of the core CCR4-NOT complex is better understood, little remains known about how various regulatory factors interact with the CCR4-NOT complex to regulate its multiple functions. It is also unclear how the CCR4-NOT complex targets specific mRNAs, which may involve a number of factors including the deadenylase module, the NOT module, poly(A)-binding protein PABP, translation factors and RNA-binding proteins [see (Collart and Panasenko, 2012) for a review]. Further structural studies will therefore be valuable in order to provide a more complete picture of the CCR4-NOT 
complex, to delineate the roles of its subunits and the interplay between them, and the association of the CCR4-NOT complex with various regulatory factors.

\section{ACKNOWLEDGMENTS}

This work was supported by the Ministry of Science and Technology 973 Project (grant number 2010CB912602) and the Ministry of Education New Century Talent Program (grant number NCET-10-0499 to Mark G. Bartlam).

\section{REFERENCES}

Albert, T. K., Hanzawa, H., Legtenberg, Y. I., De Ruwe, M. J., Van Den Heuvel, F. A. Collart, M. A., et al. (2002). Identification of a ubiquitin-protein ligase subunit within the CCR4-NOT transcription repressor complex. EMBO J. 21, 355-364. doi: 10.1093/emboj/21.3.355

Albert, T. K., Lemaire, M., Van Berkum, N. L., Gentz, R., Collart, M. A., and Timmers, H. T. (2000). Isolation and characterization of human orthologs of yeast CCR4NOT complex subunits. Nucleic Acids Res. 28, 809-817. doi: 10.1093/nar/28. 3.809

Andersen, K. R., Jonstrup, A. T., Van, L. B., and Brodersen, D. E. (2009). The activity and selectivity of fission yeast Pop2 $\mathrm{p}$ are affected by a high affinity for $\mathrm{Zn} 2+$ and Mn2+ in the active site. RNA 15, 850-861. doi: 10.1261/rna.1489409

Aslam, A., Mittal, S., Koch, F., Andrau, J. C., and Winkler, G. S. (2009). The Ccr4-NOT deadenylase subunits CNOT7 and CNOT8 have overlapping roles and modulate cell proliferation. Mol. Biol. Cell 20, 3840-3850. doi: 10.1091/mbc.E0902-0146

Badarinarayana, V., Chiang, Y. C., and Denis, C. L. (2000). Functional interaction of CCR4-NOT proteins with TATAA-binding protein (TBP) and its associated factors in yeast. Genetics 155, 1045-1054.

Bai, Y., Salvadore, C., Chiang, Y. C., Collart, M. A., Liu, H. Y., and Denis, C. L. (1999). The CCR4 and CAF1 proteins of the CCR4-NOT complex are physically and functionally separated from NOT2, NOT4, and NOT5. Mol. Cell. Biol. 19, 6642-6651.

Bartlam, M., and Yamamoto, T. (2010). The structural basis for deadenylation by the CCR4-NOT complex. Protein Cell 1, 443-452. doi: 10.1007/s13238-0100060-8

Basquin, J., Roudko, V. V., Rode, M., Basquin, C., Seraphin, B., and Conti, E. (2012). Architecture of the nuclease module of the yeast Ccr4-not complex: the Not1-Caf1-Ccr4 interaction. Mol. Cell 48, 207-218. doi: 10.1016/j.molcel.2012. 08.014

Bawankar, P., Loh, B., Wohlbold, L., Schmidt, S., and Izaurralde, E. (2013). NOT10 and C2orf29/NOT11 form a conserved module of the CCR4-NOT complex that docks onto the NOT1 N-terminal domain. RNA Biol. 10, 228-244. doi: 10.4161/rna.23018

Benson, J. D., Benson, M., Howley, P. M., and Struhl, K. (1998). Association of distinct yeast Not2 functional domains with components of Gen5 histone acetylase and Ccr4 transcriptional regulatory complexes. EMBO J. 17, 6714-6722. doi: 10.1093/emboj/17.22.6714

Bhaskar, V., Roudko, V., Basquin, J., Sharma, K., Urlaub, H., Seraphin, B., et al (2013). Structure and RNA-binding properties of the Not1-Not2-Not5 module of the yeast Ccr4-Not complex. Nat. Struct. Mol. Biol. 20, 1281-1288. doi: 10.1038/nsmb.2686

Bogdan, J. A., Adams-Burton, C., Pedicord, D. L., Sukovich, D. A., Benfield, P. A., Corjay, M. H., etal. (1998). Human carbon catabolite repressor protein (CCR4)-associative factor 1: cloning, expression and characterization of its interaction with the B-cell translocation protein BTG1. Biochem. J. 336(Pt 2), 471-481.

Boland, A., Chen, Y., Raisch, T., Jonas, S., Kuzuoglu-Ozturk, D., Wohlbold, L., et al. (2013). Structure and assembly of the NOT module of the human CCR4-NOT complex. Nat. Struct. Mol. Biol. 20, 1289-1297. doi: 10.1038/nsmb.2681

Chen, J., Chiang, Y. C., and Denis, C. L. (2002). CCR4, a $3^{\prime}-5^{\prime}$ poly(A) RNA and ssDNA exonuclease, is the catalytic component of the cytoplasmic deadenylase. EMBO J. 21, 1414-1426. doi: 10.1093/emboj/21.6.1414

Collart, M. A. (2003). Global control of gene expression in yeast by the Ccr4-Not complex. Gene 313, 1-16. doi: 10.1016/S0378-1119(03)00672-3

Collart, M. A., and Panasenko, O. O. (2012). The Ccr4 - not complex. Gene 492, 42-53. doi: 10.1016/j.gene.2011.09.033
Collart, M. A., Panasenko, O. O., and Nikolaev, S. I. (2013). The Not3/5 subunit of the Ccr4-Not complex: a central regulator of gene expression that integrates signals between the cytoplasm and the nucleus in eukaryotic cells. Cell. Signal. 25, 743-751. doi: 10.1016/j.cellsig.2012.12.018

Collart, M. A., and Struhl, K. (1993). CDC39, an essential nuclear protein that negatively regulates transcription and differentially affects the constitutive and inducible HIS3 promoters. EMBO J. 12, 177-186.

Collart, M. A., and Struhl, K. (1994). NOT1(CDC39), NOT2(CDC36), NOT3, and NOT4 encode a global-negative regulator of transcription that differentially affects TATA-element utilization. Genes Dev. 8, 525-537. doi: 10.1101/gad.8. 5.525

Deluen, C., James, N., Maillet, L., Molinete, M., Theiler, G., Lemaire, M., et al. (2002). The Ccr4-not complex and yTAF1 (yTaf(II)130p/yTaf(II)145p) show physical and functional interactions. Mol. Cell. Biol. 22, 6735-6749. doi: 10.1128/MCB.22.19.6735-6749.2002

Dominguez, C., Bonvin, A. M., Winkler, G. S., Van Schaik, F. M., Timmers, H. T., and Boelens, R. (2004). Structural model of the UbcH5B/CNOT4 complex revealed by combining NMR, mutagenesis, and docking approaches. Structure 12, 633-644. doi: 10.1016/j.str.2004.03.004

Draper, M. P., Liu, H. Y., Nelsbach, A. H., Mosley, S. P., and Denis, C. L. (1994). CCR4 is a glucose-regulated transcription factor whose leucine-rich repeat binds several proteins important for placing CCR4 in its proper promoter context. Mol. Cell. Biol. 14, 4522-4531.

Draper, M. P., Salvadore, C., and Denis, C. L. (1995). Identification of a mouse protein whose homolog in Saccharomyces cerevisiae is a component of the CCR4 transcriptional regulatory complex. Mol. Cell. Biol. 15, 3487-3495.

Dupressoir, A., Morel, A. P., Barbot, W., Loireau, M. P., Corbo, L., and Heidmann, T. (2001). Identification of four families of yCCR4- and Mg2+dependent endonuclease-related proteins in higher eukaryotes, and characterization of orthologs of yCCR4 with a conserved leucine-rich repeat essential for hCAF1/hPOP2 binding. BMC Genomics 2:9. doi: 10.1186/1471-21 64-2-9

Ezzeddine, N., Chang, T. C., Zhu, W., Yamashita, A., Chen, C. Y., Zhong, Z., etal. (2007). Human TOB, an antiproliferative transcription factor, is a poly(A)-binding protein-dependent positive regulator of cytoplasmic mRNA deadenylation. Mol. Cell. Biol. 27, 7791-7801. doi: 10.1128/MCB.01 254-07

Fabian, M. R., Cieplak, M. K., Frank, F., Morita, M., Green, J., Srikumar, T., et al. (2011). miRNA-mediated deadenylation is orchestrated by GW182 through two conserved motifs that interact with CCR4-NOT. Nat. Struct. Mol. Biol. 18, 12111217. doi: 10.1038/nsmb.2149

Fabian, M. R., Frank, F., Rouya, C., Siddiqui, N., Lai, W. S., Karetnikov, A., et al. (2013). Structural basis for the recruitment of the human CCR4-NOT deadenylase complex by tristetraprolin. Nat. Struct. Mol. Biol. 20, 735-739. doi: $10.1038 /$ nsmb.2572

Funakoshi, Y., Doi, Y., Hosoda, N., Uchida, N., Osawa, M., Shimada, I., et al. (2007). Mechanism of mRNA deadenylation: evidence for a molecular interplay between translation termination factor eRF3 and mRNA deadenylases. Genes Dev. 21, 3135-3148. doi: 10.1101/gad.1597707

Garces, R. G., Gillon, W., and Pai, E. F. (2007). Atomic model of human Rcd-1 reveals an armadillo-like-repeat protein with in vitro nucleic acid binding properties. Protein Sci. 16, 176-188. doi: 10.1110/ps.062600507

Gavin, A. C., Bosche, M., Krause, R., Grandi, P., Marzioch, M., Bauer, A., et al. (2002). Functional organization of the yeast proteome by systematic analysis of protein complexes. Nature 415, 141-147. doi: 10.1038/415141a

Halter, D., Collart, M. A., and Panasenko, O. O. (2014). The Not4 E3 Ligase and CCR4 Deadenylase play distinct roles in protein quality control. PLoS ONE 9:e86218. doi: 10.1371/journal.pone.0086218

Hanzawa, H., De Ruwe, M. J., Albert, T. K., Van Der Vliet, P. C., Timmers, H. T., and Boelens, R. (2001). The structure of the C4C4 ring finger of human NOT4 reveals features distinct from those of C3HC4 RING fingers. J. Biol. Chem. 276, 10185-10190. doi: 10.1074/jbc.M009298200

Horiuchi, M., Takeuchi, K., Noda, N., Muroya, N., Suzuki, T., Nakamura, T., et al. (2009). Structural basis for the antiproliferative activity of the TobhCaf1 complex. J. Biol. Chem. 284, 13244-13255. doi: 10.1074/jbc.M8092 50200

Ito, K., Inoue, T., Yokoyama, K., Morita, M., Suzuki, T., and Yamamoto, T. (2011a). CNOT2 depletion disrupts and inhibits the CCR4-NOT deadenylase complex 
and induces apoptotic cell death. Genes Cells 16, 368-379. doi: 10.1111/j.13652443.2011.01492.x

Ito, K., Takahashi, A., Morita, M., Suzuki, T., and Yamamoto, T. (2011b). The role of the CNOT1 subunit of the CCR4-NOT complex in mRNA deadenylation and cell viability. Protein Cell 2, 755-763. doi: 10.1007/s13238-011-1092-4

Jonstrup, A. T., Andersen, K. R., Van, L. B., and Brodersen, D. E. (2007). The 1.4-A crystal structure of the $S$. pombe Pop2p deadenylase subunit unveils the configuration of an active enzyme. Nucleic Acids Res. 35, 3153-3164. doi: 10.1093/nar/gkm178

Lau, N. C., Kolkman, A., Van Schaik, F. M., Mulder, K. W., Pijnappel, W. W., Heck, A. J., et al. (2009). Human Ccr4-Not complexes contain variable deadenylase subunits. Biochem. J. 422, 443-453. doi: 10.1042/BJ20090500

Lee, T. I., Wyrick, J. J., Koh, S. S., Jennings, E. G., Gadbois, E. L., and Young, R. A. (1998). Interplay of positive and negative regulators in transcription initiation by RNA polymerase II holoenzyme. Mol. Cell. Biol. 18, 4455-4462.

Lenssen, E., Oberholzer, U., Labarre, J., De Virgilio, C., and Collart, M. A. (2002). Saccharomyces cerevisiae Ccr4-not complex contributes to the control of Msn2pdependent transcription by the Ras/cAMP pathway. Mol. Microbiol. 43, 10231037. doi: $10.1046 / j .1365-2958.2002 .02799 . x$

Liu, H. Y., Badarinarayana, V., Audino, D. C., Rappsilber, J., Mann, M., and Denis, C. L. (1998). The NOT proteins are part of the CCR4 transcriptional complex and affect gene expression both positively and negatively. EMBO J. 17, 1096-1106. doi: 10.1093/emboj/17.4.1096

Mahadevan, S., and Struhl, K. (1990). Tc, an unusual promoter element required for constitutive transcription of the yeast HIS3 gene. Mol. Cell. Biol. 10, $4447-4455$.

Maillet, L., and Collart, M. A. (2002). Interaction between Notlp, a component of the Ccr4-not complex, a global regulator of transcription, and Dhhlp, a putative RNA helicase. J. Biol. Chem. 277, 2835-2842. doi: 10.1074/jbc.M107979200

Maillet, L., Tu, C., Hong, Y. K., Shuster, E. O., and Collart, M. A. (2000). The essential function of Not1 lies within the Ccr4-Not complex. J. Mol. Biol. 303, 131-143. doi: 10.1006/jmbi.2000.4131

Malvar, T., Biron, R. W., Kaback, D. B., and Denis, C. L. (1992). The CCR4 protein from Saccharomyces cerevisiae contains a leucine-rich repeat region which is required for its control of ADH2 gene expression. Genetics 132, 951-962.

Mauxion, F., Chen, C. Y., Seraphin, B., and Shyu, A. B. (2009). BTG/TOB factors impact deadenylases. Trends Biochem. Sci. 34, 640-647. doi: 10.1016/j.tibs.2009.07.008

Mauxion, F., Preve, B., and Seraphin, B. (2013). C2ORF29/CNOT11 and CNOT10 form a new module of the CCR4-NOT complex. RNA Biol. 10, 267-276. doi: 10.4161/rna.23065

Miller, J. E., and Reese, J. C. (2012). Ccr4-Not complex: the control freak of eukaryotic cells. Crit. Rev. Biochem. Mol. Biol 47, 315-333. doi: 10.3109/10409238.2012.667214

Mittal, S., Aslam, A., Doidge, R., Medica, R., and Winkler, G. S. (2011). The Ccr4a (CNOT6) and Ccr4b (CNOT6L) deadenylase subunits of the human Ccr4-Not complex contribute to the prevention of cell death and senescence. Mol. Biol. Cell 22, 748-758. doi: 10.1091/mbc.E10-11-0898

Morita, M., Oike, Y., Nagashima, T., Kadomatsu, T., Tabata, M., Suzuki, T., et al. (2011). Obesity resistance and increased hepatic expression of catabolismrelated mRNAs in Cnot3+/- mice. EMBO J. 30, 4678-4691. doi: 10.1038/emboj. 2011.320

Morita, M., Suzuki, T., Nakamura, T., Yokoyama, K., Miyasaka, T., and Yamamoto, T. (2007). Depletion of mammalian CCR4b deadenylase triggers elevation of the p27Kip1 mRNA level and impairs cell growth. Mol. Cell. Biol. 27, 4980-4990. doi: 10.1128/MCB.02304-06

Muhlrad, D., and Parker, R. (2005). The yeast EDC1 mRNA undergoes deadenylation-independent decapping stimulated by Not2p, Not4p, and Not5p. EMBO J. 24, 1033-1045. doi: 10.1038/sj.emboj.7600560

Nasertorabi, F., Batisse, C., Diepholz, M., Suck, D., and Bottcher, B. (2011). Insights into the structure of the CCR4-NOT complex by electron microscopy. FEBS Lett. 585, 2182-2186. doi: 10.1016/j.febslet.2011.05.071

Oberholzer, U., and Collart, M. A. (1998). Characterization of NOT5 that encodes a new component of the Not protein complex. Gene 207, 61-69. doi: 10.1016/S0378-1119(97)00605-7

Panasenko, O. O., and Collart, M. A. (2011). Not4 E3 ligase contributes to proteasome assembly and functional integrity in part through Ecm29. Mol. Cell. Biol. 31, 1610-1623. doi: 10.1128/MCB.01210-10
Petit, A. P., Wohlbold, L., Bawankar, P., Huntzinger, E., Schmidt, S., Izaurralde, E., et al. (2012). The structural basis for the interaction between the CAF1 nuclease and the NOT1 scaffold of the human CCR4-NOT deadenylase complex. Nucleic Acids Res. 40, 11058-11072. doi: 10.1093/nar/gks883

Russell, P., Benson, J. D., and Denis, C. L. (2002). Characterization of mutations in NOT2 indicates that it plays an important role in maintaining the integrity of the CCR4-NOT complex. J. Mol. Biol. 322, 27-39. doi: 10.1016/S00222836(02)00707-6

Sandler, H., Kreth, J., Timmers, H. T., and Stoecklin, G. (2011). Not1 mediates recruitment of the deadenylase Caf1 to mRNAs targeted for degradation by tristetraprolin. Nucleic Acids Res. 39, 4373-4386. doi: 10.1093/nar/gkr011

Siddiqui, N., Mangus, D. A., Chang, T. C., Palermino, J. M., Shyu, A. B., and Gehring, K. (2007). Poly(A) nuclease interacts with the C-terminal domain of polyadenylate-binding protein domain from poly(A)-binding protein. J. Biol. Chem. 282, 25067-25075. doi: 10.1074/jbc.M701256200

Temme, C., Zhang, L., Kremmer, E., Ihling, C., Chartier, A., Sinz, A., et al. (2010). Subunits of the Drosophila CCR4-NOT complex and their roles in mRNA deadenylation. RNA 16, 1356-1370. doi: 10.1261/rna.2145110

Thore, S., Mauxion, F., Seraphin, B., and Suck, D. (2003). X-ray structure and activity of the yeast Pop2 protein: a nuclease subunit of the mRNA deadenylase complex. EMBO Rep. 4, 1150-1155. doi: 10.1038/sj.embor.7400020

Tucker, M., Valencia-Sanchez, M. A., Staples, R. R., Chen, J., Denis, C. L., and Parker, R. (2001). The transcription factor associated Ccr4 and Caf1 proteins are components of the major cytoplasmic mRNA deadenylase in Saccharomyces cerevisiae. Cell 104, 377-386. doi: 10.1016/S0092-8674(01)00225-2

Viswanathan, P., Chen, J., Chiang, Y. C., and Denis, C. L. (2003). Identification of multiple RNA features that influence CCR4 deadenylation activity. J. Biol. Chem. 278, 14949-14955. doi: 10.1074/jbc.M211794200

Wahle, E., and Winkler, G. S. (2013). RNA decay machines: deadenylation by the Ccr4-not and Pan2-Pan3 complexes. Biochim. Biophys. Acta 1829, 561-570. doi: 10.1016/j.bbagrm.2013.01.003

Wang, H., Morita, M., Yang, X., Suzuki, T., Yang, W., Wang, J., et al. (2010). Crystal structure of the human CNOT6L nuclease domain reveals strict poly(A) substrate specificity. EMBO J. 29, 2566-2576. doi: 10.1038/emboj.2010.152

Winkler, G. S. (2010). The mammalian anti-proliferative BTG/Tob protein family. J. Cell. Physiol. 222, 66-72. doi: 10.1002/jcp.21919

Winkler, G. S., and Balacco, D. L. (2013). Heterogeneity and complexity within the nuclease module of the Ccr4-Not complex. Front. Genet. 4:296. doi: $10.3389 /$ fgene. 2013.00296

Yamashita, A., Chang, T. C., Yamashita, Y., Zhu, W., Zhong, Z., Chen, C. Y., etal. (2005). Concerted action of poly(A) nucleases and decapping enzyme in mammalian mRNA turnover. Nat. Struct. Mol. Biol. 12, 1054-1063. doi: 10.1038/nsmb1016

Yang, X., Morita, M., Wang, H., Suzuki, T., Yang, W., Luo, Y., et al. (2008). Crystal structures of human BTG2 and mouse TIS21 involved in suppression of CAF1 deadenylase activity. Nucleic Acids Res. 36, 6872-6881. doi: 10.1093/nar/gkn825

Zheng, D., Ezzeddine, N., Chen, C. Y., Zhu, W., He, X., and Shyu, A. B. (2008). Deadenylation is prerequisite for P-body formation and mRNA decay in mammalian cells. J. Cell Biol. 182, 89-101. doi: 10.1083/jcb.200801196

Zwartjes, C. G., Jayne, S., Van Den Berg, D. L., and Timmers, H. T. (2004). Repression of promoter activity by CNOT2, a subunit of the transcription regulatory Ccr4not complex. J. Biol. Chem. 279, 10848-10854. doi: 10.1074/jbc.M311747200

Conflict of Interest Statement: The authors declare that the research was conducted in the absence of any commercial or financial relationships that could be construed as a potential conflict of interest.

Received: 28 February 2014; accepted: 26 April 2014; published online: 16 May 2014. Citation: Xu K, Bai Y, Zhang A, Zhang Q and Bartlam MG (2014) Insights into the structure and architecture of the CCR4-NOT complex. Front. Genet. 5:137. doi: 10.3389/fgene.2014.00137

This article was submitted to Non-Coding RNA, a section of the journal Frontiers in Genetics.

Copyright $\odot 2014 \mathrm{Xu}$, Bai, Zhang, Zhang and Bartlam. This is an open-access article distributed under the terms of the Creative Commons Attribution License (CC BY). The use, distribution or reproduction in other forums is permitted, provided the original author(s) or licensor are credited and that the original publication in thisjournal is cited, in accordance with accepted academic practice. No use, distribution or reproduction is permitted which does not comply with these terms. 